\title{
Research on intelligent control system of active equalization for charging station of electric vehicle
}

\author{
Zhiyuan Zhang \\ Dept. of Electronic Information and Automation Chongqing University of Technology, \\ Chongqing 400050 P.R.China \\ zzy@cqut.edu.cn
}

Keywords: charging station; power batteres ;active equalization ; fuzzy control

\begin{abstract}
In order to improve the quality of electric vehicle battery,this paper presented an intelligent active equalization system for electric vehicle charging station. It applied equalization mode of release and supplementary to balance battery energy,and applied centralize - decentralize structure to build the system. Inductance and capacitor is applied to store up energy and transfer energy for feedback of energy. Fuzzy control technique is applied to control process in system . Simulation results show that the system can realize to balance voltage of batteres when charging or no- charging, and meet the requirement of electric vehicle charging station.
\end{abstract}

\section{Introduction}

The development of electric vehicles is important way to improve the competitiveness of automobile industry and to reduce environmental pollution . How to build a charging stations of electric vehicle, it is the bottleneck problem in application . The electric vehicle charging station have fast and slow charging function currently. With the development of electric vehicles, have the power car battery equalization function, will become important demand of power station.As a result of each single battery body performance may not be completely consistent inevitably lead to single cell difference increases, seriously affecting the service life of the battery, the impact on traffic safety. So balanced control must become an indispensable function and key technology.

\section{Structure and mode of Balanced system}

Conditions of charging station is difference with Electric vehicle ${ }^{[1]}$. Equalization system of ways and the design of the structure must be completed by the battery management system in the charging station. The charging station should have a multi - level control structure (see Figure 1),

The central module of the host computer controls several local units by CAN bus. Goup (the multiple battery) is controlled by local unit .The function of the battery charging and battery balancing , and data acquisition of the battery , and capacity computing, display and communication functiois completed by local module .

\section{way of Voltage balance and energy transfer}

There are two ways, it is passive equalization and active equalization. Voltage balance must be completed together with the charging process If charging unit does not work , the equalizer does not work too.As battery is in using process for long time,charge is not equalization in the of battery, so the differences between charge quantity of battery will become large increasingly . Active equalization completely dependent on the charging process ,Whether it is in the state of charge ,or discharge status ,or pending state,the voltage balance can be achieved.Obviously, active equalization is the best way. Because battery capacity and power electric vehicles is great ,resistor can not be used to achieve a balanced. Energy feedback equalizer Is designed for a Battery pack.

\section{Topological structure of Dispersion and centralize}

In this paper the combination of dispersion and concentrated way is used, i.e. a centralized structure on battery pack is used( Figure 1). Each battery pack uses an equalization 
controller,can be suitably simplify the control structure. It can to meet the different voltage requirements of electric vehicles to multiple groups. Inductor-capacitor as the energy storage element,the energy was balanced in using energy of this battery group .Whether the static and dynamic state of battery, it can be achieved to balance.This is an efficient active-balanced structure.

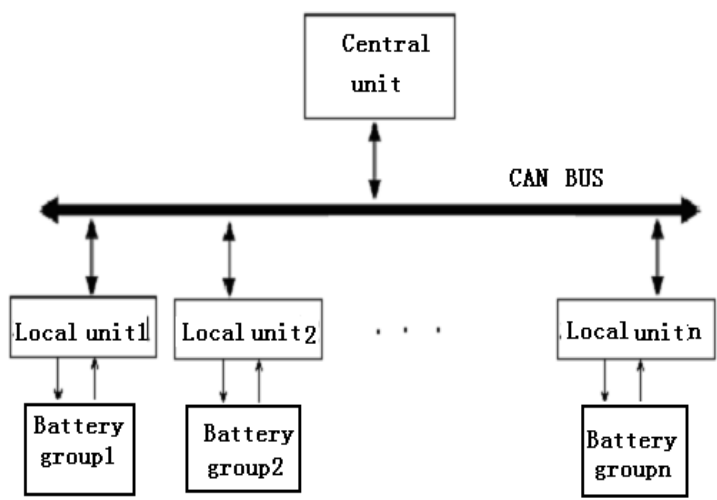

Fig.1 Structure of the charging system

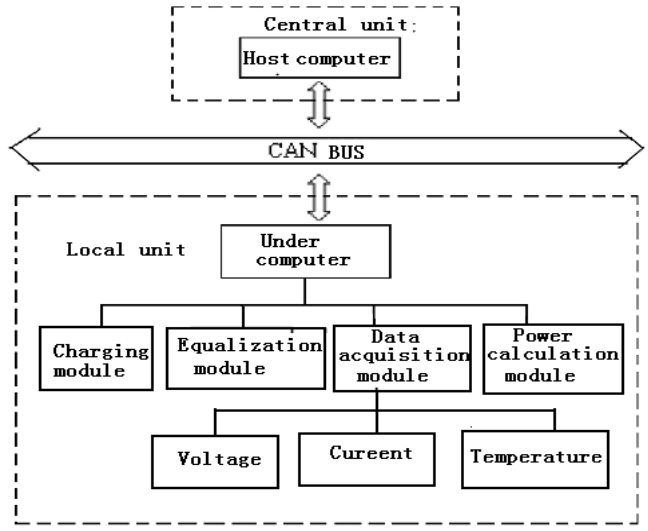

Fig.2 Structure of the local control cell

Each using a controller, It is convenient for realization of the management and control with network .The local control module uses an embedded computer with a high performance microprocessor or DSP chip to control charging unit, equalization unit and the data acquisition unit , as well as other auxiliary unit. various functional unit with higher cost performance MCU for intelligent control ( Figure 2).High-performance PC or workstation on the management system of charging station contorls local modules by the high-speed bus.

The equalization systemt with the controller, PWM generator and a logic control circuit, and an equalizer main circuit to can be to complete the equalization of a group of cells in local control module, the principle shown in Figure 3 . The system can either be done separately battery equalization, also can to work together with the main charging circuit, and equalization is completed during the charging process

\section{Dsign of equalization circuit and controller}

The balanced main circuit uses the energy storage element (such as transformers, capacitors, inductors) and switching circuit, to achieve a reasonable flow of energy ${ }^{[2-4]}$. In this article, a DC switching circuit as equalization main circuit is used. the switch-off, changing the energy of the batteries, capacitors and inductors, in order to achieve the mutual transfer of electricity in the battery pack to complete the battery pack voltage balancefunction. When the switch is turned on or off ,energy of batteries, and capacitors, and inductors is changed. The energy of the battery pack was balanced.

The battery pack has three single-battery B1, B2, and B3.The equalization circuit is composited by power device T1, T2, T3, and Tr, the diodes D1, D2, and D3, Dr. Capacitor Cr and inductor L1, L2, L3 and Lr is the reserve energy element. The PWM signal of the microcontroller control the opening time of the switching circuit, the logic circuit control power devices on and off.

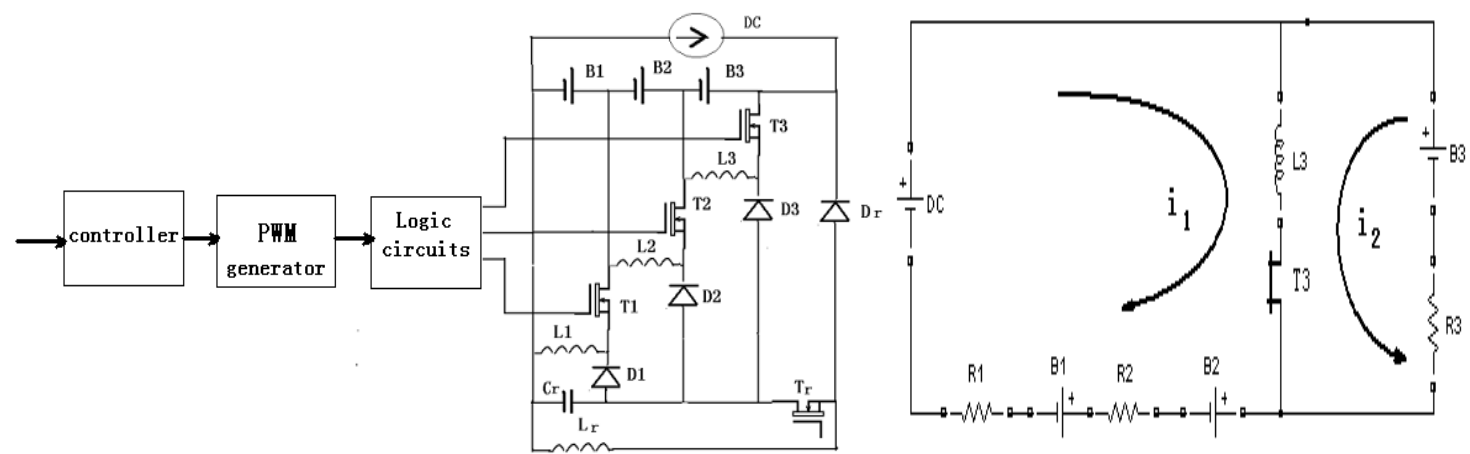


Fig.3 Principle diagram of equalization circuit

\section{Energy transfer on static mode}

The circuit have two mode of static and dynamic, the battery is not charging on static. Activate the equalizer, the energy transfer from the high voltage of the single battery,Bi, energy transfer processes are as follows:

$\mathrm{VBi}$ is voltage for the $\mathrm{i}$-th cell , $\mathrm{V}$ e is he average voltage for the battery pack.if $\mathrm{VBi}>\mathrm{Ve}$, then $\mathrm{Ti}$ is opened,and cell $\mathrm{Bi}$ excess power is transferred to the inductance $\mathrm{Li}$, the current value through Ti:

$$
i_{t i}=\frac{V_{B i}}{L} D_{\text {on }} \quad 0<t<D_{\text {on }} T
$$

Don: the duty cycle of the PWM control signal; T: PWM control signal cycle. when Ti is off, discharge loop is composed by $\mathrm{Li}, \mathrm{Bi}+1 \ldots \mathrm{Bn}, \mathrm{Cr}, \mathrm{Di}$, $\mathrm{Li}$, so electric power on $\mathrm{Li}$ will be transferred to following cell and Cr.when the capacitor voltage VCR value exceeds the set value,Tr is off,electric powere is recharge the battery pack by Dr.

\section{Energy transfer on dynamic mode}

System is dynamically balanced mode when the battery pack is charged by an external power supply .In this case, there are two loop current (Figure 4). Energy is released from B3 with high voltage cell to L3 by i2, and cell B1,B2 were charged by i1 .Therefore, dynamic current is greater than the static, and less time(Figure 5). The i1 is determined by the formula (2), i2 is determined by the formula (3).

$$
\begin{array}{cc}
i_{1}(t)=\frac{V_{d c}(t)-V_{L}(t)-V_{T}(t)-V_{B 2}(t)-V_{B 1}(t)}{R_{2}+R_{1}} & 0<t<D_{\text {on }} T \\
i_{2}(t)=\frac{V_{B 3}(t)-V_{L}(t)-V_{T}(t)}{R_{3}} & 0<t<D_{\text {on }} T
\end{array}
$$

$\mathrm{Vdc}$ is voltage of charging power supply, $\mathrm{V}_{\mathrm{L}}$ is voltage of inductor, $\mathrm{V}_{\mathrm{T}}$ is voltage on switch -on , VB3, VB2, VB1 is the battery voltage, R3, and R2, R1 is the Internal resistance of the battery. Since the parameters of the battery pack is changed in the energy transfer process, and the charging and equalization at the same time occurs .It is a complex and dynamic process that is difficult to describe precise mathematical model.The fuzzy control method as a control strategy to control the energy release and transfer process ${ }^{[5]}$.

\section{Simulation results}

In Matlab environment, a battery model is created based on Simulink Kit .According to experimental data, the function of the relation betwenn electromotive force and the SOC of batter was obtained, In the balancing process, the change of the electromotive force can be obtained. The simulation result of dynamic equilibrium response is shown in Figure 7, the simulation .result of the static equilibrium response is shown in Figure 8.Clearly, the characteristics of the variable control is better than the characteristics of the fixed PWM duty the error is large, balanced regulation is strong, When the error is small, balanced regulation is weak.The equalizer process and the precision is the same. 


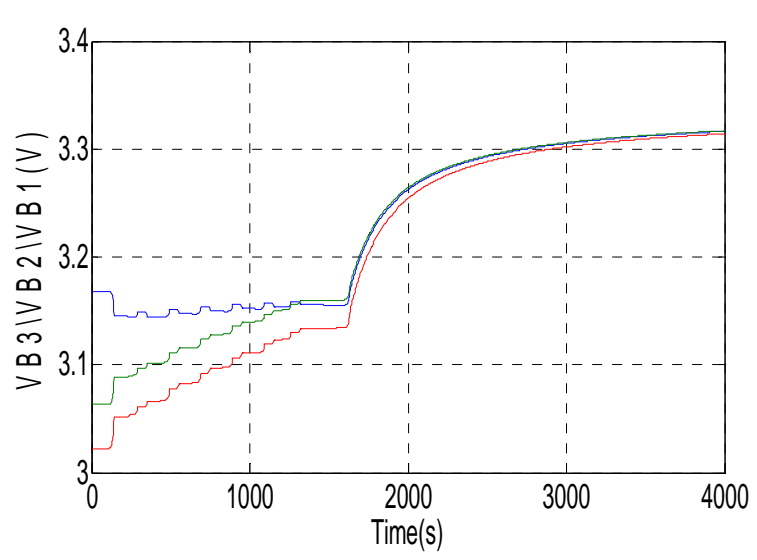

Fig.5 Voltages of batteries when charging

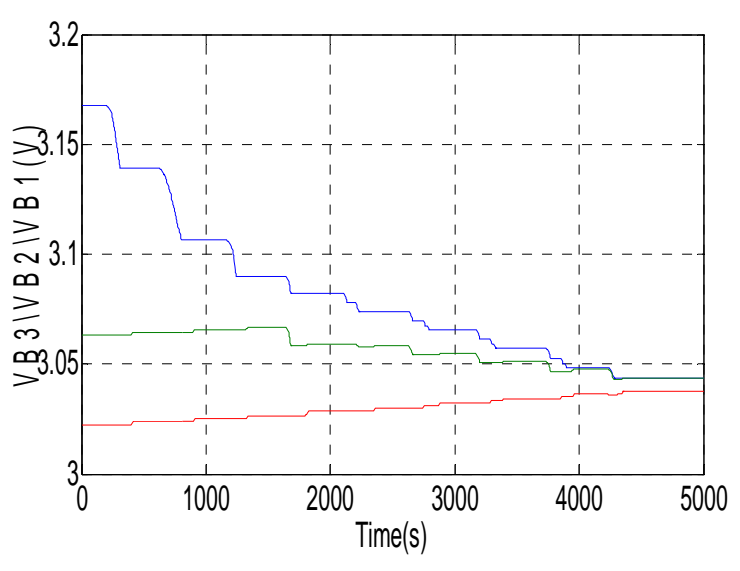

Fig.6 Voltages of batteries when no-charging

\section{Conclusion}

This paper analyzes the structure and technology of the balance control system, design a balanced way and structure for charging station of electric vehicle. the use of the packet charging and balanced way, battery combination, which can meet the power requirements of different voltage valuesUsing grouped manner of charging and balancing, and easily battery combination, can meet the power with different voltage value.At the same time,energy utilization efficiencycan be improved with the active balancing and energy feedback. As practical control algorithm is designed for automatic control, so the balanced response process is optimized. The simulation result shows that the system is feasible, provides a valuable way of charging station construction forelectric vehicle.

\section{References}

[1] Wu Youyu , Liang Hong,A Study on Equalization Charging for EV Traction Battery,Automotive Engineering,2004 (Vol. 26) No. 4:382-385

[2] Teng letian, Design charging station of electric vehicle,Beijin:China Electric Power Press, 2009.5 : 115-173

[3] XU Ai-guo, XIE Shao-jun, LIU Xiao-bao,Dynamic Voltage Equalization Technique for Series Connected Ultra-capacitors,Proceedings of the CSEE,Vol.30 No.12 Apr.25, 2010:111-115

[4] Yuang-Shung Lee, Guo-Tian Chen. ZCS Bi-directional DC-to-DC Converter Application in Battery qualization for Electric Vehicles[J]. IEEE,2004.

[5] Zhang zhiyuan,Wan peilin,Design of fuzzy control system in driving control of electric vehile ,Electric Machines and Control,2005 (Vol.9) No.3:203-206 